La dernière ligne du tableau périodique de Mendeleïev est maintenant complète et l'élément le plus lourd, l'oganesson, compte 118 protons. II s'agit là de la limite atteinte avec les technologies actuelles, mais pas la limite d'existence des éléments chimiques.

\section{Une nouvelle génération}

d'instruments est en

construction pour aller

plus loin et pour synthétiser en plus grand nombre les derniers éléments découverts, afin de pouvoir étudier leurs propriétés.

Nous remercions Jean-Charles Thomas pour sa relecture attentive.

\title{
À la recherche de l'ultime atome
}

\author{
La dernière ligne du tableau de Mendeleïev \\ est complétée. La physique des éléments \\ superlourds aborde de nouveaux défis.
}

Christelle Stodel $^{(1)}$ (christelle.stodel@ganil.fr), David Boilley ${ }^{(1,2)}$, Julien Piot ${ }^{(1)}$ et Dieter Ackermann ${ }^{(1)}$ (1) GANIL (Grand Accélérateur National d'lons Lourds), CNRS/IN2P3 et CEA/DRF, Bvd Henri Becquerel, 14076 Caen Cedex 5 (2) Université Caen-Normandie (UNICAEN), 14032 Caen Cedex 5

\section{La limite d'existence des éléments chimiques}

La dernière ligne du tableau périodique des éléments est maintenant remplie : les derniers entrants s'appellent nihonium ${ }^{(a)}$ $(\mathrm{Nh})$, moscovium $^{(\mathrm{b})}(\mathrm{Mc})$, tennessine $\mathrm{e}^{(\mathrm{c})}(\mathrm{Ts})$

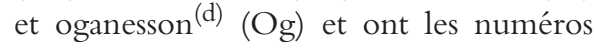
atomiques respectifs $Z=113,115,117$ et 118 [1] (fig. 1). Le tennessine est dans la colonne des halogènes qui ont une terminaison en -ine en anglais, et l'oganesson dans celle des gaz rares dont le nom se termine par -on, à l'exception de l'hélium. Il s'agit, bien évidemment, des éléments les plus lourds connus actuellement. Ils ont été formés lors d'une collision entre deux ions lourds et se sont rapidement désintégrés par émission radioactive alpha.
Les entrées précédentes, le flerovium (Fl, $Z=114)$ et le livermorium $(\mathrm{Lv}, Z=116)$ datent de 2012. Il y a eu le copernicium (Cn, $Z=112)$ en 2010. Y en aura-t-il d'autres dans un proche avenir ? La réponse est négative, car il n'y a plus de nouvel élément formé en attente de validation. Nous avons probablement atteint la limite de ce que l'on peut synthétiser avec les instruments actuels, mais pas la limite d'existence des éléments. Une nouvelle génération d'instruments est en construction pour aller plus loin et pour étudier plus en détail les derniers entrés dans la classification périodique, grâce à une plus grande statistique.

Du point de vue de la physique atomique, la limite d'existence des atomes a été évaluée à $Z=172$. Les modèles relativistes qui décrivent leur structure électronique en

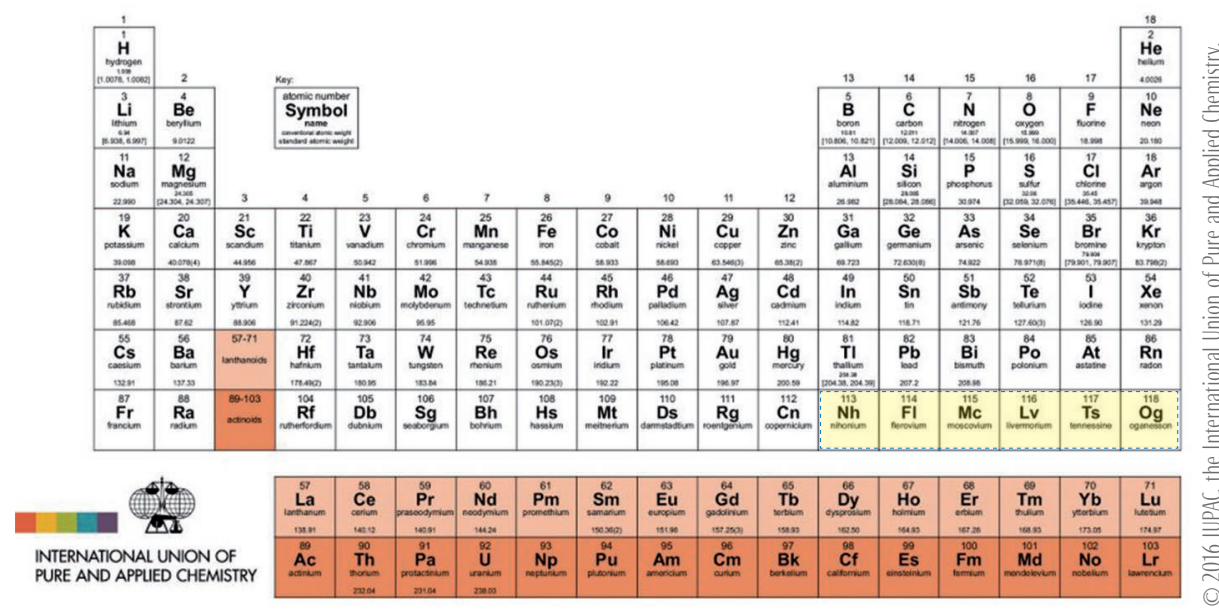

1. Version complète du tableau périodique des éléments chimiques, validée par l'IUPAC en 2016. Les derniers éléments découverts ( $(=113$ à 118) sont colorés en jaune.. 


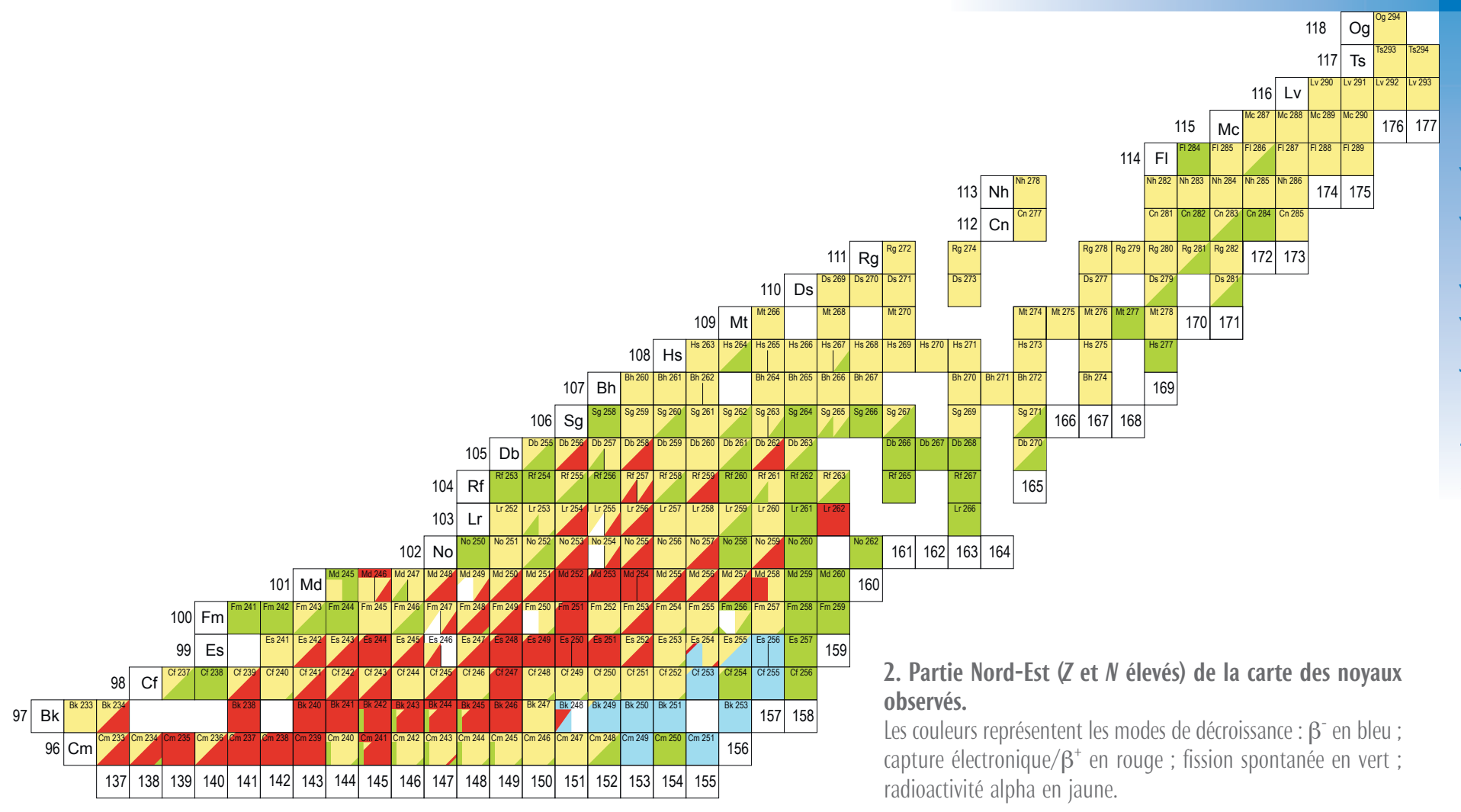

prenant en compte l'électrodynamique quantique (QED) montrent qu'au-delà les orbitales électroniques pénètrent dans le noyau et l'énergie de l'électron 1s devient négative. On en est loin, car le noyau correspondant n'existe pas et aucun modèle actuel ne prévoit son existence. C'est donc bien du côté du noyau atomique et de la physique nucléaire qu'il faut aller chercher la limite d'existence des éléments chimiques. Comme les noyaux naturels les plus lourds, tous les noyaux artificiels sont radioactifs. Mais leur durée de vie doit être suffisamment longue $\left(>10^{-14} \mathrm{~s}\right)$ pour qu'ils puissent former des atomes, avec leur cortège électronique. Une autre limite d'existence vient de la fission spontanée. L'énergie de liaison qui maintient les nucléons ensemble, permet de quantifier la stabilité des noyaux : elle est assez bien décrite par le modèle dit de la goutte liquide. Face à la déformation du noyau, ce sont les termes dus à la répulsion coulombienne entre les protons et à la tension de surface, liée à l'interaction forte entre les nucléons, qui dominent. Quand on augmente la taille des noyaux, le terme de surface grandit moins vite que le terme coulombien et, au-delà de $Z=100$, la barrière de potentiel qui empêche que le noyau se casse en deux spontanément (barrière de fission) disparaît. C'est ainsi que John A. Wheeler a proposé une première limite d'existence des noyaux et donc des éléments chimiques en 1955, sans pour autant donner un nombre de protons précis. Il introduit aussi le terme " noyaux superlourds".

C'est toutefois sans compter les effets quantiques : à l'instar des électrons autour du noyau, les nucléons sont ordonnés en niveaux d'énergie discrets, également appelés couches. Dans le cas des électrons des atomes, les couches entièrement remplies correspondent aux gaz rares. De même, lorsque les protons ou les neutrons remplissent totalement leurs propres couches, les noyaux correspondants sont dits " magiques » (ou " doublement magiques" lorsque les couches sont entièrement remplies aussi bien par les protons que par les neutrons). Les noyaux correspondants sont plus stables. Le noyau doublement magique le plus lourd connu dans la nature est le plomb-208, avec 82 protons et 126 neutrons. Quels sont les noyaux magiques suivants ? Si les modèles s'accordent sur le fait qu'il devrait y avoir une zone de la carte des noyaux (fig. 2) avec un regain de stabilité significatif lié à la magicité, sa localisation n'est pas la même pour toutes les prévisions. Pour les neutrons, c'est $N=184$ qui est généralement proposé, mais pour les protons, cela va de $Z=114$ à $Z=126$ suivant les modèles.

La demi-vie de certains isotopes superlourds atteint des dizaines de minutes (par exemple le ${ }^{267} \operatorname{Rf}(Z=104)$ avec une demi-vie de $1,1 \mathrm{~h}$ ), soit 16 ordres de grandeur plus long que celui prédit sans la stabilisation due aux effets quantiques. Les demi-vies de décroissance alpha d'autres isotopes sont représentées sur la figure 3 en fonction du nombre de neutrons. Elles augmentent jusqu'à un maximum situé à $N=162$, identifié comme un nombre

\〉

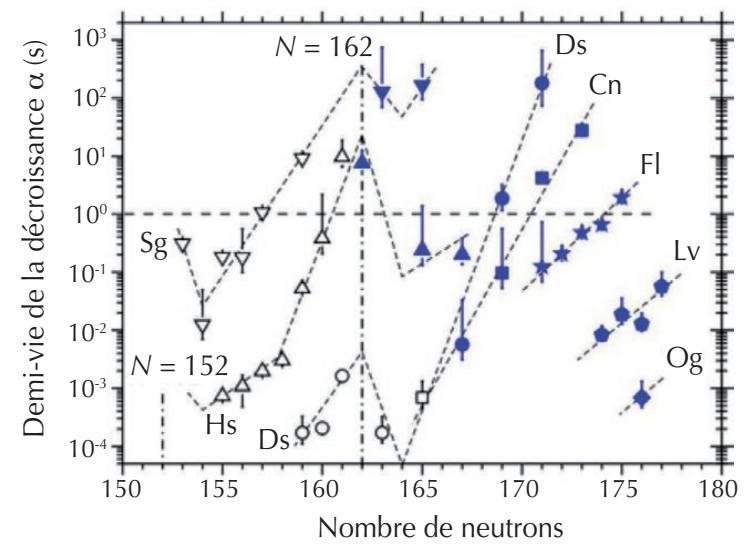

3. Demi-vies partielles des décroissances alpha des isotopes dont le nombre de protons est pair, à partir du seaborgium $(Z=106)$. D’après Yu.Ts. Oganessian et al. [2]. 


\section{>>}

magique en neutrons pour noyaux déformés. Ensuite, une nouvelle augmentation semble indiquer l'approche d'une prochaine couche fermée située au-delà de $N=180$.

En physique nucléaire, le terme "noyaux superlourds " désigne les isotopes dont le nombre de charge est supérieure à $Z=100$. Les chimistes, quant à eux, préferent adopter le terme "superlourd " pour les éléments à partir du rutherfordium $(Z=104)$ où la couche $5 \mathrm{f}$ électronique est complète.

\section{Nouveaux éléments, nouvelles questions}

Les interrogations sur la matière sont très anciennes. La conception d'un objet insécable qui constituerait toute matière, l'atome, date au moins de l'époque antique (voir encadré 1). Compléter le tableau périodique de Mendeleiev est donc d'une grande importance, même si les éléments les plus récents ne survivent pas longtemps et que les trouver sur Terre est ainsi mission impossible. En revanche, ils pourraient apparaitre lors d'évènements extrêmement violents dans l'Univers comme les supernovas ou lors de collisions entre étoiles à neutrons.

Une fois l'existence d'un nouvel élément vérifiée, il est tout aussi important d'étudier ses propriétés physiques et chimiques. Par exemple, les effets relativistes influent sur la structure atomique des éléments de la sixième ligne du tableau de Mendeleïev $(Z=55$ à 86). Ils deviennent primordiaux pour ceux de la septième ligne $(Z=87$ à 118) et donc pour les éléments superlourds. On peut alors se demander si le principe actuel de périodicité gouvernant les groupes et périodes du tableau de Mendeleïev sont toujours valides pour les derniers entrés.

Du côté du noyau, les nombres magiques ont tendance à s'effacer dans la région des superlourds, pour être remplacés par des zones magiques où plusieurs noyaux proches bénéficient d'un regain de stabilité lié aux effets quantiques. Alors que les noyaux «magiques " plus légers sont préférentiellement sphériques, des indices permettent de supposer que certains noyaux superlourds sont plutôt déformés, acquérant ainsi un surcroît de stabilité. On pourrait également expliquer leur relative stabilité par une configuration interne des nucléons en agrégats. Tout cela doit être vérifié expérimentalement.

\section{La synthèse des éléments superlourds}

Si les premiers éléments transuraniens ont été formés par capture neutronique suivie de décroissances radioactives (voir encadré 2), les noyaux superlourds sont principalement synthétisés à l'aide de réactions nucléaires dites de fusion-évaporation. Pour cela, on envoie, grâce à un accélérateur, un noyau projectile sur une cible mince de matière. On choisit une énergie suffisante pour combattre la répulsion coulombienne qui est de l'ordre de 5-6 MeV par nucléon, soit une vitesse du projectile de l'ordre de $10 \%$ de la célérité de la lumière. Les réactions sont alors diverses : elles vont de la simple diffusion élastique sans modification des noyaux cible et projectile, ou diffusion Rutherford, à des réactions de fusion complète.

Dans ce dernier cas, le noyau composé ainsi formé est dans un état excité et a une énergie résiduelle, dite d'excitation, qui doit être évacuée. Par ailleurs, cette énergie d'excitation a tendance à laver les effets de couches qui conferent aux noyaux superlourds un regain de stabilité. Le noyau se comporte alors comme une simple goutte liquide et aura majoritairement tendance à se séparer de nouveau en deux fragments, autrement dit fissionner. Ce processus, qui dure environ $10^{-21} \mathrm{~s}$, est trop rapide pour qu'un cortège électronique ait le temps de se former autour du noyau éphémère afin d'obtenir un atome complet et donc pré- tendre avoir formé un nouvel élément chimique.

Toutefois, dans une très faible minorité des cas, le noyau composé va émettre un ou plusieurs neutrons qui emportent chacun une quantité définie d'énergie, jusqu'à ce que le noyau résiduel soit suffisamment " refroidi " pour pouvoir être stabilisé par les effets de couches quantiques. On qualifie ce processus de fusion-évaporation.

Décrire précisément la réaction reste un défi, car le mécanisme de formation differe de celui observé pour des noyaux plus légers. La fusion est entravée, c'est-à-dire que la probabilité de fusion observée est bien plus faible que ce que pourraient prédire les modèles habituels. L'origine de ce phénomène est bien comprise : deux noyaux légers en contact vont fusionner automatiquement grâce à la tension de surface. Une forme en $\infty$ a une énergie de surface beaucoup plus élevée que celle d'un noyau composé sphérique. Mais pour les noyaux plus lourds, la répulsion coulombienne est telle que même au contact, les deux noyaux vont avoir tendance à se reséparer plutôt que de fusionner : on appelle ce processus la quasi-fission. Augmenter l'énergie de la collision afin d'avoir une impulsion suffisante pour fusionner n'est pas une option, car le noyau superlourd composé est extrêmement fragile. Pour accroître ses chances de survie, il est préférable que l'énergie résiduelle du noyau ainsi formé soit la plus faible possible. Ces deux tendances opposées expliquent

\section{Histoire de la notion d'élément chimique}

Alors que l'hypothèse atomique, au sens indivisible, a été émise par Leucippe puis Démocrite, il s'agissait pour ces auteurs d'une spéculation philosophique plutôt que d'un résultat issu d'une démarche scientifique. L'idée fut contestée par leur contemporain Aristote dont la représentation du monde, basée sur quatre substances élémentaires (l'eau, l'air, la terre et le feu), a prévalu pendant plus de 2100 ans.

$\mathrm{Au} 17^{\mathrm{e}}$ siècle, la "science moderne " se développe avec des techniques de chimie et permet de ressortir des cartons le concept d'élément chimique introduit par Robert Boyle en 1664 ; suite à ses expériences sur les gaz, il l'avait défini par un « nombre limité de corps simples et primitifs ne pouvant être fabriqués par aucun autre corps ». À la fin du $18^{\mathrm{e}}$ siècle, Antoine-Laurent Lavoisier précise alors la définition de l'élément chimique par l'impossibilité de le décomposer à l'aide de moyens chimiques en substances plus simples. Ensuite, John Dalton, en 1808, introduit l'idée que le poids atomique est une caractéristique utile d'un élément chimique. Cela a motivé les chimistes à chercher des relations entre le poids atomique et les propriétés chimiques des éléments, et a ainsi conduit Dimitri Mendeleïev en 1869 à ordonner périodiquement les éléments et à prédire I'existence de certains.

Suite à la découverte des rayonnements X par Roentgen et aux travaux de H.G.J. Moseley en 1913, on a préféré caractériser un élément par sa charge atomique ou son numéro atomique $Z$ plutôt que par son poids atomique. 


\section{Historique de la découverte des transuraniens}

encadré 2

L'uranium $(Z=92)$ est le dernier élément chimique existant à l'état naturel sur Terre, sa demi-vie étant de l'ordre de grandeur de l'âge de cette dernière. Au-delà, les éléments sont formés artificiellement.

Dans les années 1940, les premiers éléments transuraniens ont été produits par décroissance $\beta^{-} d^{\prime}$ isotopes riches en neutrons d'uranium puis de plutonium, créés par la capture successive de neutrons dans les réacteurs nucléaires (et plus tard dans les explosions nucléaires). Cependant cette méthode ne peut permettre d'accéder aux éléments au-delà du fermium $(Z=100)$, car la décroissance alpha ou la fission spontanée deviennent prépondérants. La production d'éléments plus lourds n'est possible que par des réactions de fusion-évaporation complète. De 1944 à 1974, les éléments du curium $(Z=96)$ au seaborgium $(Z=106)$ furent ainsi produits par irradiation de cibles d'actinides avec des projectiles légers appropriés, disponibles et faciles à accélérer.

Cependant, l'énergie d'excitation de plusieurs dizaines de MeV du noyau composé formé par ces réactions favorise la fission et limite la formation des noyaux superlourds.

En 1974, Yuri Oganessian et ses collaborateurs ont expérimenté la fusion à partir de noyaux fortement liés (plus que les actinides) de ${ }^{208} \mathrm{~Pb}$ et ${ }^{209} \mathrm{Bi}$ avec les projectiles adaptés; cette réaction de fusion froide permet au noyau composé d'être faiblement excité et ainsi de se « refroidir » par l'évaporation de seulement un ou deux neutrons. Cette stratégie a permis à l'équipe de Dubna en Russie de produire un nouvel isotope du seaborgium en 1974 et à l'équipe SHIP de GSI, à Darmstadt en Allemagne, de poursuivre la synthèse des éléments $Z=107$ à 112 de 1981 à 1996 . Néanmoins, la probabilité de fusion de deux noyaux diminue fortement en fonction de la charge totale du noyau composé. L'équipe de RIKEN à Wakô au Japon a relevé le défi en poursuivant patiemment ces réactions de fusion froide avec la technique parfaitement établie et a synthétisé l'élément 113 (3 événements en 550 jours de faisceau entre 2004 et 2012).

Dans les années 2000, l'équipe russe a développé des faisceaux intenses de ${ }^{48} \mathrm{Ca}$ (noyau naturel doublement magique avec 20 protons et 28 neutrons) permettant également d'obtenir une énergie d'excitation du noyau composé suffisamment basse pour assurer sa survie, tout en utilisant des cibles d'actinides. Grâce à cette combinaison riche en neutrons, on se rapproche de l'îlot de stabilité prédit, où les noyaux ont une haute barrière de fission leur conférant une plus grande probabilité de survie. C'est ainsi que l'équipe de Dubna a synthétisé les éléments $Z=114$ à 118 .

les très faibles sections efficaces - ou proportions de réactions gagnantes - pour les réactions de fusion-évaporation.

Dans la pratique, deux approches ont été mises en œuvre : la "fusion froide", qui correspond à la fusion suivie par l'évaporation d'un voire deux neutrons, et la "fusion chaude " où plusieurs neutrons sont émis par le noyau composé avant de former un élément superlourd. Dans le premier cas on privilégie la probabilité de survie, et dans le deuxième celle de fusion.

D'un point de vue expérimental, comme dans toute expérience de physique nucléaire, il faut réussir à séparer les évènements d'intérêt, excessivement rares dans le cas des superlourds, des autres voies de réaction. On utilise pour cela un séparateur qui dévie les noyaux sans intérêt à l'aide d'un agencement de dipôles magnétiques et électriques qui sélectionnent les produits de réaction en fonction de leur masse, de leur vitesse ou de leur charge électrique. élevée. Dans le cas de l'oganesson, le plus lourd des noyaux nouvellement créés, la cible est du californium-249 $(Z=98)$ de façon à obtenir la charge $Z=118$. Mais, pour aller au-delà avec le calcium-48, il n'est plus possible techniquement de fabriquer des cibles en quantité suffisante.

\section{Identification des nouveaux éléments}

Pour pouvoir entrer dans le tableau périodique, les nouveaux éléments doivent être identifiés sans ambiguïté. Les noyaux les plus lourds ont été identifiés grâce à leurs chaînes de décroissance alpha. Quand cette chaîne de désintégration aboutit à une région connue de la charte des noyaux, il n'y a pas d'ambiguïté : il suffit alors de suivre la décroissance alpha sur plusieurs générations jusqu'à atteindre un noyau dont l'énergie de la particule émise est connue. Il faut ensuite remonter la chaîne jusqu'au noyau le plus lourd. Il arrive toutefois que la chaîne de décroissance du noyau produit ne soit connectée à aucun noyau connu. Dans ce cas, il faut utiliser d'autres méthodes pour compléter l'identification.

Dans la pratique, les particules chargées (résidus d'évaporation et particules alpha) sont identifiées par des détecteurs à semiconducteur de silicium qui mesurent l'énergie et la position d'arrêt du noyau produit grâce à des pistes verticales d'un côté et horizontales de l'autre. Seules les particules alpha détectées aux mêmes coordonnées que le point d'implantation du noyau formé sont prises en compte car leur parcours dans la matière est très court, de l'ordre de quelques dizaines de micromètres. On peut alors suivre les transformations successives du noyau produit en sélectionnant les décroissances radioactives qui ont été observées aux mêmes coordonnées et dans un intervalle de temps raisonnable. Le détecteur de silicium est entouré d'autres détecteurs qui permettent de mesurer l'émission d'électrons ou de rayons gamma et $\mathrm{X}$. Une fois que le noyau est identifié, on peut procéder à une corrélation temporelle entre le temps d'arrivée du noyau et la détection des autres particules afin de reconstruire, chronologiquement, sa désexcitation et sa décroissance radioactive.

Quand les éléments superlourds sont produits en quantité suffisante, une identification avec des techniques de radiochimie peut être effectuée sur des atomes individuels 


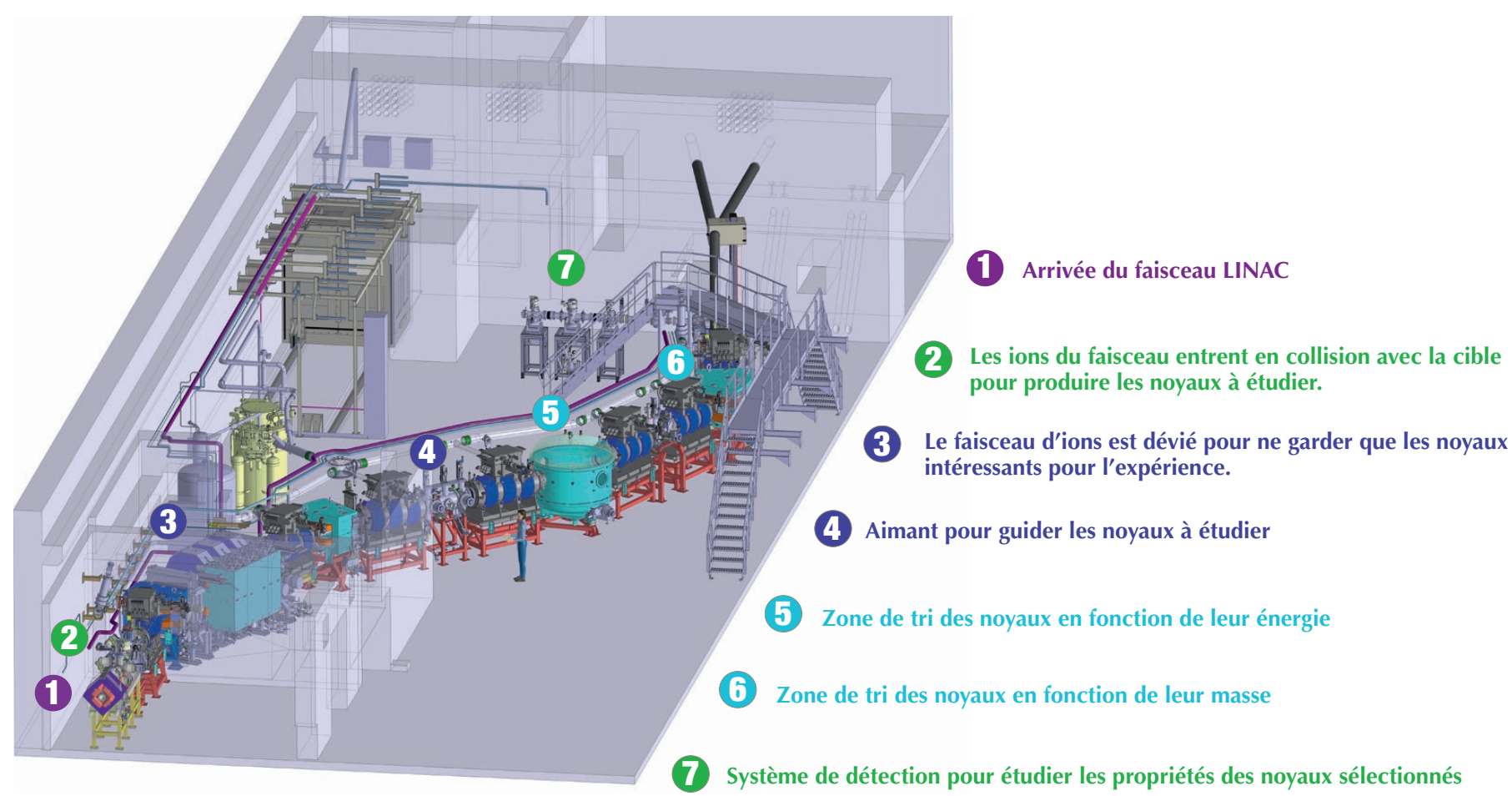

4. Schéma du spectromètre $S^{3}$ (Super Séparateur Spectromètre) auprès de Spiral2. Un équipement d'excellence pour l'étude des noyaux très lourds et superlourds.

>>

sur des temps très courts. Pour ce faire, le noyau est guidé en sortie du spectromètre par un jet de gaz porteur tel que l'hélium, vers des dispositifs de séparation chimique où le noyau est thermalisé et peut être oxydé en mélangeant le gaz avec du dioxygène. Les techniques de séparation chimique employées sont soit en phase gazeuse (thermochromatographie ou chromatographie isothermale), soit en phase liquide. La technique de thermochromatographie consiste à disposer une série de détecteurs soumis à un gradient de température. L'élément transporté par le flux gazeux est absorbé sur un détecteur à une température spécifique qui caractérisera sa volatilité. Dans la méthode isothermale, l'élément est transporté dans une colonne de température constante, au bout de laquelle il sera identifié. En mesurant, pour plusieurs températures, le temps de rétention dans la colonne et le taux en sortie, on a accès à l'enthalpie d'adsorption. Ces mesures permettent de confirmer l'analogie chimique avec les autres éléments de la même colonne du tableau de Mendeleïev.

Depuis 1985, la reconnaissance de la paternité d'un nouvel élément est la mission d'un groupe de travail (TWG $=$ Transfermium Working Group) composé de chimistes et de physiciens internationaux mandatés par les fédérations IUPAC et IUPAP (International Union of Pure and Applied Chemistry/Physics). Le critère retenu par le comité de découverte d'un élément chimique est la démonstration expérimentale, au-delà du doute raisonnable, de l'existence d'un noyau avec un numéro atomique $Z$ non identifié auparavant, survivant au moins $10^{-14} \mathrm{~s}$. Cela correspond au temps nécessaire à un noyau pour acquérir ses électrons extérieurs afin d'obtenir un "élément " avec des propriétés chimiques caractéristiques. Il n'est pas nécessaire de déterminer la valeur exacte de $Z$, seulement de prouver qu'elle est différente de toutes celles observées précédemment. Également, il n'est pas requis de connaitre la valeur exacte du nombre de masse $A$. Le groupe de travail reconnait que le terme «doute raisonnable " est vague : dans les faits, le comité préfere attendre la confirmation des résultats proclamés par leur reproductibilité, de préférence dans un autre laboratoire et/ou avec une technique différente. Cette requête n'est pas exigée lorsque les données sont de grande qualité et sont redondantes, ou lorsque les circonstances ne permettent pas une répétition de l'expérience dans des conditions raisonnables, comme pour le nihonium.

\section{Dans l'avenir}

Les principaux défis pour l'avenir sont de pouvoir augmenter la statistique des évènements afin d'aller au-delà de l'oganesson $(Z=118)$, et de produire les éléments connus en plus grand nombre afin de pouvoir étudier leurs propriétés. Partout, cela se fait en essayant de gagner en efficacité à chaque étape, de l'intensité du faisceau en amont de la cible à la séparation et à l'identification.

C'est sur ces exigences qu'a été conçu le super-spectromètre-séparateur $\mathrm{S}^{3}$ qui, couplé avec Spiral2, le futur accélérateur linéaire du GANIL, ouvrira de nouvelles opportunités de recherche sur des évènements rares dans différents domaines de la physique (fig. 4). Le programme expérimental de cette installation inclut, en particulier, l'étude des noyaux lourds et superlourds, des états fondamentaux et isomériques de noyaux légers produits par des réactions de fusion et/ou transfert de nucléons, ainsi que la spectroscopie des noyaux proches de la drip line (limite d'existence des noyaux). Le dispositif $\mathrm{S}^{3}$ comprend deux étages : le premier sépare les produits de réactions du faisceau primaire suivant la valeur de leur rigidité magnétique, et le second purifie le faisceau secondaire et sélectionne en masse les noyaux. 
Cependant, l'exploitation de faisceaux d'ions lourds plus intenses que ceux disponibles actuellement soulève de nombreux défis technologiques. On utilise déjà des cibles tournantes pour répartir l'énergie du faisceau incident et éviter leur surchauffe. Dans l'avenir, elles devront être encore plus robustes. Le spectromètre multiétage impose également l'utilisation de technologies de pointe, comme des aimants supraconducteurs.

En attendant la mise en service du spectromètre $\mathrm{S}^{3}$ dans les années à venir, la communauté française poursuit des collaborations sur cette thématique auprès d'autres accélérateurs (Dubna en Russie ou Jyväskylä en Finlande). Au GANIL, les dernières expériences effectuées sur les éléments superlourds ont utilisé la spectrométrie gamma afin d'obtenir des informations sur la structure du dubnium $(Z=105)$.

(a) En référence à Nihon, nom japonais du Japon, l'élément $Z=113$ étant le premier découvert sur le continent asiatique.

(b) Région dans laquelle a été synthétisé l'élément $Z=115$ (le dubnium $Z=105$ faisait déjà référence à la ville de Dubna où est situé le laboratoire).

(c) Pour remercier l'équipe du laboratoire d'Oak Ridge au Tennessee d'avoir fourni la cible de berkélium bombardée avec le faisceau de calcium, formant l'élément $Z=117$.

(d) En l'honneur du professeur Yuri Oganessian, pionnier dans les recherches des éléments superlourds.

(e) Unité de section efficace :

1 femtobarn $=10^{-15}$ barn $=10^{-15} \times 10^{-24} \mathrm{~cm}^{2}$; soit une chance d'observer un noyau sur $10^{21}$ projectiles.

\section{Références}

1- Communiqués de l'IUPAC

- du 30 décembre 2015

https://iupac.org/discovery-and-assignment-of-

elements-with-atomic-numbers-113-115-117-and-118/,

> du 8 juin 2016

https://iupac.org/iupac-is-naming-the-four-new-

elements-nihonium-moscovium-tennessine-and-

oganesson/

- du 30 novembre 2016

https://iupac.org/iupac-announces-the-names-ofthe-elements-113-115-117-and-118/.

2• Yu. Ts. Oganessian et al., Nuclear Physics A 944 (2015) 62-98.

Pour en savoir plus,

on peut consulter les actes du Symposium Nobel 160 :

"Chemistry and Physics of Heavy and Superheavy

Elements”, EPJ Web of Conferences, Volume 131 (2016)

www.epj-conferences.org/articles/epjconf/abs/2016/

26/contents/contents.html. 